\title{
Variables to Predict Nephrological Disease in General, and Glomerulonephritis in Particular, in Patients With Microhematuria
}

\author{
Carsten Paul Bramlage, b, e, Manuel Wallbach ${ }^{\mathrm{a}}$, David Ellenberger ${ }^{\mathrm{c}}$, Cornelia Deutsch ${ }^{\mathrm{b}}$, \\ Joan Minguet ${ }^{\mathrm{b}, \mathrm{d}}$, Katherine Helen Smith ${ }^{\mathrm{d}}$, Johanna Stock ${ }^{\mathrm{a}}$, Alina Goninski ${ }^{\mathrm{a}}$, Peter Bramlage ${ }^{\mathrm{b}}$, \\ Michael Koziolek ${ }^{\mathrm{a}}$, Gerhard Anton Mueller ${ }^{\mathrm{a}}$
}

\begin{abstract}
Background: Microhematuria (MH) is a symptom frequently leading to uncertainty as to when a nephrology referral is appropriate. Because MH may be indicative of severe kidney disorders, prompt diagnosis and potential treatment initiation can be important. We aimed to identify further variables that point at a nephrological cause, in particular of glomerulonephritis (GN), when $\mathrm{MH}$ is diagnosed.
\end{abstract}

Methods: A retrospective analysis of data acquired from patients attending a nephrology office due to $\mathrm{MH}$ was performed. Demographic information and diagnostic tests were evaluated in order to identify factors that were associated with a nephrological cause.

Results: Patients with MH $(\mathrm{n}=805)$ as indicated by a urine stick analysis were included. Of these, MH was confirmed by urine sediment analysis in 543 patients (67.5\%). Of those, $48.3 \%$ had a nephrological cause, including $12.4 \%$ with GN and $2.9 \%$ with rapid progressive GN (RPGN). A urine dipstick finding of $\geq 250$ erythrocytes per microliter, microalbuminuria and elevated leukocytes increased the probability of having a GN to $62.4 \%$. Furthermore, the presence of microalbuminuria, GFR $<60 \mathrm{~mL} / \mathrm{min}$, history of hypertension and diabetes mellitus increased the probability for all nephrological causes to $95.4 \%$.

Conclusion: There are a number of factors available that help to assess the need for a nephrology referral in patients with microhematuria.

Keywords: Microhematuria; Kidney disease; Diagnostic; General practice; Urinalysis

Manuscript accepted for publication March 17, 2017

aDepartment of Nephrology and Rheumatology, Georg-August-University of Gottingen, Gottingen, Germany

bInstitute of Pharmacology and Preventive Medicine (IPPMED), Cloppenburg, Germany

'Institute of Medical Statistics, Georg-August-University of Gottingen, Gottingen, Germany

${ }^{\mathrm{d}}$ Institute for Research and Medicine Advancement (IRM), Terrassa, Spain ${ }^{e}$ Corresponding Author: Carsten Paul Bramlage, Department of Nephrology and Rheumatology, Georg-August University of Gottingen, Robert-Koch-Str. 40,37075 Gottingen, Germany. Email: c.bramlage@med.uni-goettingen.de

doi: https://doi.org/10.14740/jocmr2993w

\section{Introduction}

Microhematuria $(\mathrm{MH})$ is commonly defined as the presence of at least three red blood cells per field of view under a highpower lens during analysis of a properly obtained urine specimen [1]. Usually asymptomatic, it is mostly an accidental finding that is considered to be of little importance unless patients are at an advanced age and are at risk of cancer [2]. Therefore, there is uncertainty as to the extent of workup needed [3-5].

The source of MH may be located anywhere along the urinary tract, with a wide variety of possible causes. These include benign reasons such as mild trauma or infections [6], but also malignancies [7]. Furthermore, a proportion of patients presenting with $\mathrm{MH}$ will have a nephrological condition that requires intervention in order to delay progression to end-stage renal disease $(E S R D)$ and dialysis $[1,8]$. The presence of dysmorphic red blood cells, proteinuria, cellular casts, and/or renal insufficiency indicates a need for further investigation to establish if there is a glomerular cause [1].

It is apparent from the literature that there is a high level of uncertainty surrounding the usefulness and accuracy of $\mathrm{MH}$ to predict serious nephrological disorders. This is particularly true among the majority of office-based physicians, which rarely have the means to perform urine sediment microscopy, and thus insufficient information to make a decision on the need for a nephrology referral $[9,10]$. Clarification of this situation is essential for enabling accurate and rapid diagnosis.

We therefore carried out a retrospective evaluation of patients presenting with $\mathrm{MH}$ attending our nephrology office. The aim of the analysis was to identify factors that are routinely determined by the office-based physician and increase the likelihood of a nephrological cause of MH. Such information would improve the identification of patients that would benefit from early referral to a nephrologist, allowing for rapid diagnosis and initiation of appropriate treatment [8].

\section{Methods}

\section{Study design}

We carried out a retrospective analysis of data from patients 
Table 1. Patient Characteristics

\begin{tabular}{|c|c|}
\hline & Mean \pm SD (range) or $\mathrm{n} / \mathrm{N}(\%)$ \\
\hline \multicolumn{2}{|l|}{ Epidemiology $(\mathrm{N}=805)$} \\
\hline Age (years) & $56.3 \pm 18.1(6-91)$ \\
\hline Patients $\geq 40$ years & $665 / 805(82.6 \%)$ \\
\hline Sex (male) & $389 / 805(48.3 \%)$ \\
\hline BMI $\left(\mathrm{kg} / \mathrm{m}^{2}\right)$ & $27.8 \pm 6.0(12.6-64.7)$ \\
\hline \multicolumn{2}{|l|}{$\mathrm{CV}$ risk factors $(\mathrm{N}=543)$} \\
\hline $\mathrm{SBP}(\mathrm{mm} \mathrm{Hg})$ & $144.7 \pm 23.5(90-227)$ \\
\hline DBP (mm Hg) & $82.4 \pm 14.4(50-145)$ \\
\hline History of arterial hypertension & $359 / 543(66.1 \%)$ \\
\hline Diabetes mellitus, type 1 or 2 & $86 / 543(15.8 \%)$ \\
\hline Hyperlipidemia & $178 / 543(32.8 \%)$ \\
\hline Current smoker & $132 / 543(24.3 \%)$ \\
\hline \multicolumn{2}{|l|}{ Kidney function $(\mathrm{N}=543)$} \\
\hline eGFR (mL/min) & $62.1 \pm 34.1(2-186)$ \\
\hline $\mathrm{eGFR}<90 \mathrm{~mL} / \mathrm{min}$ & $375 / 502(74.1 \%)$ \\
\hline $\mathrm{eGFR}<60 \mathrm{~mL} / \mathrm{min}$ & $236 / 502(47.1 \%)$ \\
\hline Microalbuminuria $>20 \mathrm{mg} / \mathrm{L}$ & $255 / 372(68.5 \%)$ \\
\hline \multicolumn{2}{|l|}{ Signs of urinary tract infection $(\mathrm{N}=543)$} \\
\hline Leukocytes in urine test strip $\geq 25 / \mu \mathrm{L}$ & $229 / 543(42.2 \%)$ \\
\hline Nitrite in urine & $37 / 543(6.8 \%)$ \\
\hline Chronic infection of the urinary tract & $53 / 543(9.8 \%)$ \\
\hline
\end{tabular}

Epidemiological data were taken from the whole cohort $(N=805)$, whereas the other data $(N=543)$ were taken only from patients with confirmed $\mathrm{MH}$ ( $\geq 3$ erythrocytes per high power field of view in urine sediment analysis). BMI: body mass index; CV: cardiovascular; SBP: systolic blood pressure; DBP: diastolic blood pressure; eGFR: estimated glomerular filtration rate.

who presented at our nephrology office between 1998 and 2014. All patients with MH in a prior urine test strip analysis were included. No further inclusion or exclusion criteria were applied.

\section{Documentation}

For all patients, demographic information was collected, along with the result of urine test strip analysis. Further data were recorded for those patients that tested positive for $\mathrm{MH}$ in the urine sediment analysis, being defined as $\geq 3$ erythrocytes per visual field under a high power lens. A full medical history was taken, including the intake of relevant drugs (anticoagulation and NSAID), and additional diagnostic testing was documented. The estimated glomerular filtration rate (eGFR) was calculated using the CKD-EPI formula, and signs of a urinary tract infection (leukocyturia and nitrite) were recorded. Coagulation abnormalities (prothrombin time (PTT), Quick, international normalized ratio (INR), and thrombocytes) and systemic inflammation were evaluated.

The potential sources of the MH were taken from the patient files, which included the results of all diagnostic tests, including the kidney biopsy if one had been performed.

The study was conducted according to the principles of the Declaration of Helsinki.

\section{Statistical analysis}

Data were presented as means with standard deviations (SDs) and range or absolute values with percentages. Boosted regression was used to preselect variables affecting the likelihood of a nephrological cause (or glomerulonephritis (GN) specifically). The association analysis was based on patients with all necessary data available $(\mathrm{n}=339$ complete cases $)$.

Logistic regression with Firth's penalised likelihood approach was used in order to reduce potential bias caused by low event rates. Univariate and multivariate effect estimates (OR) along with 95\% confidence intervals (CIs) were given. Furthermore effect measures on the log OR were dichotomised to create a risk score. This model was then recalibrated based on the total risk score sums to get appropriate values for the probability of an event, with the area under the curve (AUC) as a goodness-of-fit measure. A P-value of $<0.05$ was considered to be statistically significant. All analyses were performed us- 
Table 2. Characteristics of Hematuria

\begin{tabular}{|c|c|}
\hline & $\mathrm{n} / \mathbf{N}(\%)$ \\
\hline \multicolumn{2}{|c|}{ Erythrocytes in urine stick analysis (per $\mu \mathrm{L}$ ) } \\
\hline$<10$ & $78 / 805(9.7 \%)$ \\
\hline$\geq 10-49$ & $371 / 805(46.1 \%)$ \\
\hline$\geq 50-249$ & $188 / 805(23.4 \%)$ \\
\hline$\geq 250$ & $167 / 805(20.8 \%)$ \\
\hline Macroscopic hematuria & $37 / 805(4.6 \%)$ \\
\hline Chronic hematuria & $20 / 805(2.5 \%)$ \\
\hline Confirmed MH (urine sediment) ${ }^{\dagger}$ & $543 / 805(67.5 \%)$ \\
\hline
\end{tabular}

Data from the urine test strip analysis were collected at the first visit to the nephrologist $(\mathrm{N}=805)$. ${ }^{\dagger}$ Confirmed hematuria is defined as patients that had $\geq 3$ erythrocytes per high power field of view in urine sediment analysis.

ing the software Statistica 12 (Statsoft, Hamburg, Germany) and R (R Foundation, Vienna, Austria).

\section{Results}

\section{Patient characteristics and MH}

A total of 805 patients that presented at the nephrology office due to an office-based physician diagnosis of $\mathrm{MH}$ were included in the study. The mean age of this population was 56.3 \pm 18.1 years (range: 6 - 91 years) (Table 1 ). At the time of the first nephrology visit, urine test strip analysis identified $90.3 \%$ of the 805 patients with $\geq 10$ erythrocytes per microliter, and the remaining proportion with fewer than 10 erythrocytes per microliter. Macroscopic hematuria was documented for $4.6 \%$ of the 805 patients and chronic haematuria for $2.5 \%$ (Table 2).

$\mathrm{MH}$ was confirmed by urine sediment analysis in 543 $(67.5 \%)$ patients. Thus, a total of 262 patients $(32.5 \%)$ were determined to have transient $\mathrm{MH}$ or false positive urine test strip analysis (Table 2).

For the patients with $\mathrm{MH}$ confirmed by the urine sediment analysis, $66.1 \%(359 / 543)$ of patients had a history of arterial hypertension, $32.8 \%(178 / 543)$ hyperlipidemia and $15.8 \%$ $(86 / 543)$ diabetes mellitus (type 1 or 2$)$.

The eGFR was found to be below the normal value of 90 $\mathrm{mL} / \mathrm{min}$ in $74.1 \%$ of the patients with $47.1 \%(236 / 502)$ having an eGFR value below $60 \mathrm{~mL} / \mathrm{min}$ (Table 1).

\section{Underlying causes of $\mathrm{MH}$}

A potential nephrological cause of $\mathrm{MH}$ was identified for $48.3 \%(262 / 543)$ of patients (Table 3$)$. The underlying disease was determined to be GN in $12.4 \%(68 / 543)$ of patients, with this diagnosed as RPGN in 2.9\% (16/543). Interstitial nephritis, generally attributed to medication use, was found in $6.1 \%$ $(33 / 543)$ of patients. High proportions of patients were found to have a first diagnosis of hypertensive $(26.5 \% ; 144 / 543)$ or
Table 3. Potential Sources of MH Identified in the Nephrology Office (Multiple Selections Are Possible)

\begin{tabular}{|cl}
\hline & n/N (\%) \\
\hline Nephrological disease (all) & $262 / 543(48.3 \%)$ \\
\hline Glomerulonephritis (all) & $68 / 543(12.4 \%)$ \\
\hline Rapid progressive glomerulonephritis & $16 / 543(2.9 \%)$ \\
\hline Interstitial nephritis & $33 / 543(6.1 \%)$ \\
\hline Congenital kidney disease & $15 / 543(2.8 \%)$ \\
\hline Diabetic nephropathy & $51 / 543(9.4 \%)$ \\
\hline Hypertensive nephropathy & $144 / 543(26.5 \%)$ \\
\hline Other kidney diseases* & $16 / 543(2.9 \%)$ \\
\hline Urological source & $113 / 543(20.8 \%)$ \\
\hline Infection of the urinary tract & $55 / 543(10.1 \%)$ \\
\hline Pyelonephritis & $10 / 543(1.8 \%)$ \\
Coagulation abnormality & $64 / 541(11.8 \%)$ \\
Vitamin K antagonist/NOAC use & $44 / 543(8.1 \%)$ \\
\hline Anti-platelet drug use ${ }^{\dagger}$ & $97 / 543(17.9 \%)$ \\
\hline NSAID use & $66 / 543(12.2 \%)$ \\
\hline Systemic inflammation & $124 / 543(22.8 \%)$ \\
\hline Number of potential sources of MH & \\
\hline 1 & $147 / 543(27.1 \%)$ \\
\hline 2 & $127 / 543(23.4 \%)$ \\
\hline Unclear diagnosis, benign MH & $76 / 543(14.0 \%)$ \\
\hline & $19 / 543(3.5 \%)$ \\
\hline & $174 / 543(32.0 \%)$ \\
\hline
\end{tabular}

NOAC: non-vitamin-K oral anticoagulant; NSAID: non-steroidal antiinflammatory drug. *Includes multiple myeloma and renal amyloidosis; multiple myeloma; scleroderma; and suspected or non-proven glomerulonephritis. \#Thrombocytopenia, increased INR, or elevated prothrombin time. †Includes aspirin, clopidogrel, dipyridamol, ticlopidine. ${ }^{\ddagger} \mathrm{CRP}>$ $5 \mathrm{mg} / \mathrm{L}$ or ESR $>20 \mathrm{~mm} / \mathrm{h}$ or leukocytes $>10 / \mathrm{hL}$.

diabetic $(9.4 \% ; 51 / 543)$ nephropathy.

Only $27.1 \%(147 / 543)$ of patients had a single potential cause, with multiple reasons particularly common for the patients with renal diseases that are not normally associated with $\mathrm{MH}$, such as hypertensive and diabetic nephropathy.

The most frequent concomitant cause of $\mathrm{MH}$ was urological $(20.8 \% ; 113 / 543)$, with $54.0 \%$ also presenting with a nephrological disorder. Further concomitant reasons were systemic inflammation $(22.8 \% ; 124 / 543)$, urinary tract infections $(10.1 \% ; 55 / 543)$ and coagulation abnormality $(11.8 \%$; $64 / 541)$, with $8.1 \%(44 / 543)$ being treated with anticoagulants or anti-platelet agents $(17.9 \%$; 97/543) (Table 3$)$.

\section{Relationship between MH and a diagnosis of kidney dis- ease}

Kidney disease was diagnosed in $262(48.3 \%)$ of the 543 patients that were found to have $\mathrm{MH}$ in the urine sediment analy- 


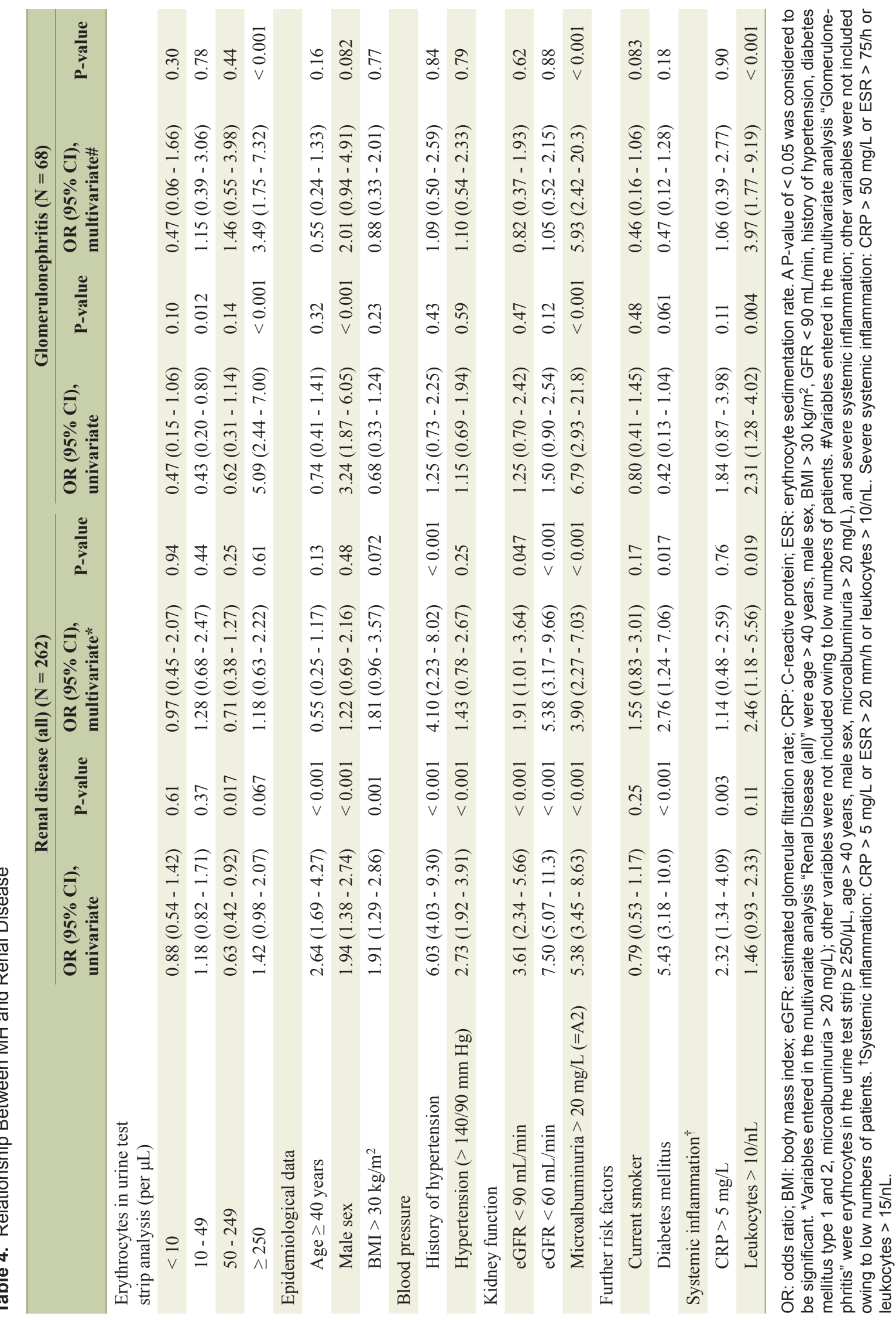


sis. In univariate analysis, a patient age of $\geq 40$ years was associated with a nephrological disease, as was male gender and a BMI of $>30 \mathrm{~kg} / \mathrm{m}^{2}$ (Table 4). In addition, a history of hypertension, blood pressure $>140 / 90 \mathrm{mmHg}$, eGFR impairment, diabetes mellitus (type 1 and 2), and elevated systemic inflammation were associated with kidney disease in our cohort.

Factors associated with a patient that had a nephrological cause of $\mathrm{MH}$ were determined by selecting variables found to be statistically significant in multivariable logistic regression, while also being available in the daily physician office. The variables identified were a history of hypertension (OR: 3.98; 95\% CI: 2.16 - 7.77; P $<0.001)$, eGFR $<60 \mathrm{~mL} / \mathrm{min}(\mathrm{OR}$ : 5.17; 95\% CI: $3.05-9.21 ; \mathrm{P}<0.001)$, the presence of microalbuminuria (OR: 3.76; 95\% CI: $2.20-6.75$; P $<0.001$ ), and diabetes mellitus (type 1 or 2 ; OR: 2.84 ; $95 \%$ CI: $1.29-7.24$; $\mathrm{P}=0.013$ ). The number of these indicators that are present can be regarded as a risk score for having a nephrological disease $(\mathrm{AUC}=0.850)$, with an estimated probability of $95.4 \%$ whenever all four indicators are present (Fig. 1).

In the univariate analysis (328 complete cases), the likelihood of gomerulonephritis specifically was found to be higher for patients with a urine test strip results of $\geq 250$ erythrocytes/ $\mu \mathrm{L}$ (OR: 5.09; 95\% CI: $2.44-7.01 ; \mathrm{P}<0.001$ ), those with microalbuminuria (OR: 6.79; 95\% CI: 2.93 - 21.8; $\mathrm{P}<0.001$ ), and those with elevated leukocytes $(\geq 10 / \mu \mathrm{L}$; OR: $2.31 ; 95 \%$ CI: $1.28-4.02 ; \mathrm{P}=0.004)$. The cut-off of $\geq 10$ leukocytes/ $\mu \mathrm{L}$ was chosen from the $80^{\text {th }}$ percentile of the data as a cutoff value of $\geq 11 / \mu \mathrm{L}$ yielded too few elevated cases (Table 4 ). The quantity of these three indicators that are present can be regarded as a risk score for having $\mathrm{GN}(\mathrm{AUC}=0.773)$, with an estimated probability of $62.8 \%$ in the case of all three indicators being present (Fig. 1).

\section{Discussion}

In order to identify factors that may indicate the need for a referral to a nephrologist for an individual presenting with $\mathrm{MH}$, we retrospectively analyzed data from patients attending our nephrology office. Certain factors were found to be associated with a greater likelihood of the presence of an underlying nephrological cause of the patient's MH. From the collated data, we calculated probabilities of nephrological disease in general and GN specifically, which could be used by an officebased physician when determining the necessity for a nephrology referral.

GN was a potential source of $\mathrm{MH}$ for $12.4 \%$ of patients, with $2.9 \%$ of these cases determined to be rapid progressive GN, a condition that can result in ESRD and a need for dialysis within a few weeks of its development. It is for these patients that a prompt consultation with a nephrologist is the most important. A urine test strip finding of $\geq 250$ erythrocytes $/ \mu \mathrm{L}$, microalbuminuria, and elevated leukocyte count were all associated with GN, with a calculated probability of this condition being present of $62.8 \%$ if all three indicators were found. These could therefore be important parameters to indicate the presence of a serious nephrological disorder to the office-based physician, possibly even before a decrease in kid- ney function/eGFR becomes apparent. All of these variables are readily available to the office-based physician.

Interestingly, a high proportion of patients with $\mathrm{MH}$ were diagnosed as having hypertensive or diabetic nephropathy. These glomerulopathies are not normally associated with $\mathrm{MH}$, thus, it is likely that these patients had an additional source. Indeed, approximately $40 \%$ of the patients included in our analysis had their $\mathrm{MH}$ attributed to more than one potential cause. This includes the use of anticoagulants or anti-platelet agents, infections and urological diseases. Therefore, although hypertensive or diabetic nephropathy is unlikely to be the sole cause of the $\mathrm{MH}$, the presence of hypertension or diabetes should indicate to the office-based physicians that a nephrology referral may be warranted.

It should be noted that hypertensive nephropathy is overrepresented in our cohort. While few of these patients had a kidney biopsy, Perkowska-Ptasinska et al. showed a low prevalence of hypertension-related lesions in the biopsies they performed on their nephrology patients, a high proportion of whom had hypertension [11]. Therefore, in patients presenting with $\mathrm{MH}$ and elevated blood pressure, other renal diseases have to be ruled out

The presence of dysmorphic erythrocytes or cellular casts is a reliable indicator of a glomerular cause of the $\mathrm{MH}$ [1214]. However, many nephrological conditions present with erythrocytes of unaltered morphology [15]. In addition, office-based physicians often do not have the facilities to perform urine sediment microscopy to make a decision regarding the need for a nephrology referral [10]. Thus, we decided to include all patients with a renal cause of $\mathrm{MH}$, independent of its characteristics, in the statistical analysis and development of the probability scores. In order to aid the physician in their decision-making process, we statistically determined the individual risks associated with parameters that are routinely available in general clinical practice. Microalbuminuria, an eGFR below $60 \mathrm{~mL} / \mathrm{min}$, a history of hypertension, and the presence of diabetes mellitus type 1 or 2 were found to be highly associated with kidney disease. The probability of a nephrological disorder increased with the number of these indicators that were present, reaching approximately $95 \%$ when all four were found. Whilst we do not go so far as to recommend a cut-off point for a nephrology referral, this system provides useful guideline for the office-based physician. Validation of the model in a large cohort of patients displaying $\mathrm{MH}$ in a clinical practice setting may provide an estimation of an appropriate risk level at which a patient should be referred to a nephrologist.

\section{Limitations}

One limitation to the present analysis is its retrospective nature. In addition, only patients with $\mathrm{MH}$ were included and there was no comparison to patients without $\mathrm{MH}$. This analysis was based on the data of a single office rather than being a multi-center study. Furthermore, there are no data available from office-based physicians that may have seen these patients prior to the nephrology visit. Such information would have allowed for analysis of the appropriateness of current referral 

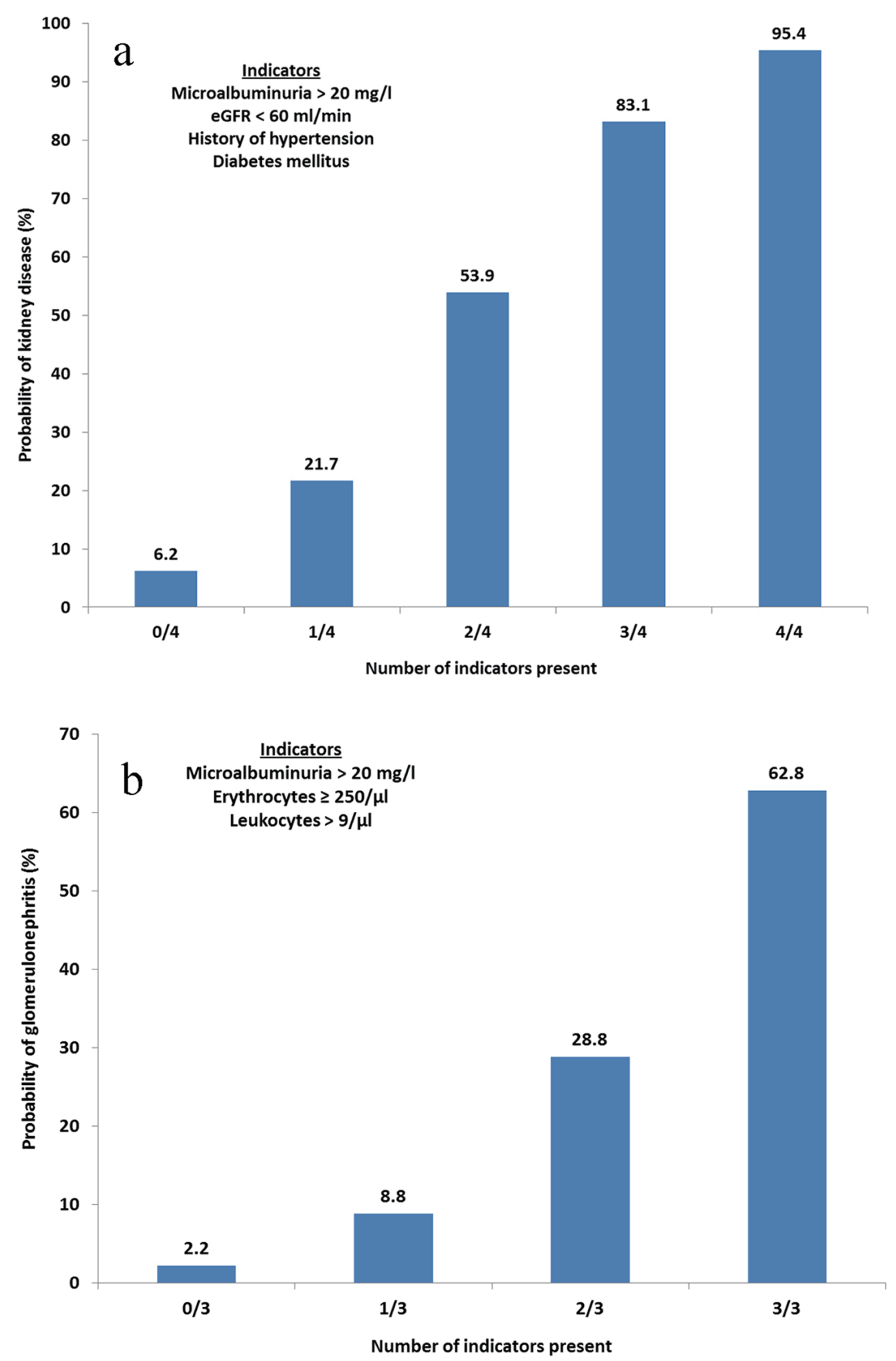

Figure 1. (a) Probability of kidney disease based on clinical findings. Calculated probabilities of kidney disease based on factors found to be significant in the multivariate logistic regression. Any combination of indicators can be present. eGFR, estimated glomerular filtration rate. (b) Probability of glomerulonephritis based on clinical findings. Calculated probabilities of glomerulonephritis based on factors found to be significant in the multivariate logistic regression. Any combination of indicators can be present. Erythrocyte concentration determined by urine stick analysis.

practices and adherence to expert guidelines.

\section{Conclusions}

In a cohort of patients attending a nephrology clinic due to a finding of $\mathrm{MH}$, a number of factors were found to be associated with the presence of the condition, including hypertension, diabetes mellitus, microalbuminuria, and a reduced eGFR. Furthermore, a urine dipstick finding of $\geq 250$ erythrocytes per microliter, microalbuminuria, and elevated leukocytes were independently associated with the presence of GN specifically. 
In order to aid office-based physicians to determine when a nephrology referral is necessary for a patient with $\mathrm{MH}$, we calculated probabilities of a nephrological disease being present, based on variables routinely available in clinical practice.

\section{Acknowledgments}

We thank Katrin Bramlage and Laura Brockmann for their help in data sampling.

\section{Conflicts of Interest}

None.

\section{References}

1. Davis R, Jones JS, Barocas DA, Castle EP, Lang EK, Leveillee RJ, Messing EM, et al. Diagnosis, evaluation and follow-up of asymptomatic microhematuria (AMH) in adults: AUA guideline. J Urol. 2012;188(6 Suppl):2473-2481.

2. Elias K, Svatek RS, Gupta S, Ho R, Lotan Y. High-risk patients with hematuria are not evaluated according to guideline recommendations. Cancer. 2010;116(12):29542959.

3. Jung H, Gleason JM, Loo RK, Patel HS, Slezak JM, Jacobsen SJ. Association of hematuria on microscopic urinalysis and risk of urinary tract cancer. J Urol. 2011;185(5):1698-1703.

4. Madeb R, Golijanin D, Knopf J, Davis M, Feng C, Fender A, Stephenson L, et al. Long-term outcome of patients with a negative work-up for asymptomatic microhematuria. Urology. 2010;75(1):20-25.

5. Ogasawara M, Aoki K, Matsuura E, Kunimatsu M, Ohkubo I, Galli M, Sasaki M, et al. Anticardiolipin an- tibodies in patients with pregnancy loss induce factor Xa production in the presence of beta 2-glycoprotein I. Am J Reprod Immunol. 1995;34(5):269-273.

6. Jaffe JS, Ginsberg PC, Gill R, Harkaway RC. A new diagnostic algorithm for the evaluation of microscopic hematuria. Urology. 2001;57(5):889-894.

7. Kaufman DS, Shipley WU, Feldman AS. Bladder cancer. Lancet. 2009;374(9685):239-249.

8. Smart NA, Dieberg G, Ladhani M, Titus T. Early referral to specialist nephrology services for preventing the progression to end-stage kidney disease. Cochrane Database Syst Rev. 2014;(6):CD007333.

9. De Coster C, McLaughlin K, Noseworthy TW. Criteria for referring patients with renal disease for nephrology consultation: a review of the literature. J Nephrol. 2010;23(4):399-407.

10. Kelly JD, Fawcett DP, Goldberg LC. Assessment and management of non-visible haematuria in primary care. BMJ. 2009;338:a3021.

11. Perkowska-Ptasinska A, Deborska-Materkowska D, Bartczak A, Stompor T, Liberek T, Bullo-Piontecka B, Wasinska A, et al. Kidney disease in the elderly: biopsy based data from 14 renal centers in Poland. BMC Nephrol. 2016;17(1):194.

12. Crop MJ, de Rijke YB, Verhagen PC, Cransberg K, Zietse $R$. Diagnostic value of urinary dysmorphic erythrocytes in clinical practice. Nephron Clin Pract. 2010;115(3):c203212.

13. Wollin T, Laroche B, Psooy K. Canadian guidelines for the management of asymptomatic microscopic hematuria in adults. Can Urol Assoc J. 2009;3(1):77-80.

14. Kohler H, Wandel E, Brunck B. Acanthocyturia - a characteristic marker for glomerular bleeding. Kidney Int. 1991;40(1):115-120.

15. Heine GH, Sester U, Girndt M, Kohler H. Acanthocytes in the urine: useful tool to differentiate diabetic nephropathy from glomerulonephritis? Diabetes Care. 2004;27(1):190-194. 